\title{
A Study of I-Function of Several Complex Variables
}

\author{
Prathima Jayarama, ${ }^{1,2}$ Vasudevan Nambisan Theke Madam, ${ }^{3}$ \\ and Shantha Kumari Kurumujji ${ }^{2,4}$ \\ ${ }^{1}$ Department of Mathematics, Manipal Institute of Technology, Manipal, Karnataka 576104, India \\ ${ }^{2}$ SCSVMV, Sri Jayendra Saraswathi Street, Enathur, Kanchipuram, Tamil Nadu 631561, India \\ ${ }^{3}$ Department of Mathematics, College of Engineering, Trikaripur, Kerala 670307, India \\ ${ }^{4}$ Department of Mathematics, P.A. College of Engineering, Mangalore, Karnataka 574153, India
}

Correspondence should be addressed to Prathima Jayarama; pamrutharaj@yahoo.co.in

Received 27 June 2013; Revised 5 September 2013; Accepted 23 September 2013; Published 27 January 2014

Academic Editor: Alberto Cardona

\begin{abstract}
Copyright ( 2014 Prathima Jayarama et al. This is an open access article distributed under the Creative Commons Attribution License, which permits unrestricted use, distribution, and reproduction in any medium, provided the original work is properly cited.

The aim of this paper is to introduce a natural generalization of the well-known, interesting, and useful Fox H-function into generalized function of several variables, namely, the I-function of " $r$ " variables. For $r=1$, we get the I-function introduced and studied by Arjun Rathie (1997) and, for $r=2$, we get I-function of two variables introduced very recently by ShanthaKumari et al. (2012). Convergent conditions, elementary properties, and special cases have also been given. The results presented in this paper generalize the results of $\mathrm{H}$-function of " $r$ " variables available in the literature.
\end{abstract}

\section{Introduction}

In 1997, Rathie introduced the generalization of the wellknown Fox's H-function [1] which has very recently found interesting applications in wireless communication [2-4]. Motivated by the I-function, very recently Shantha Kumari, Nambisan, and Rathie introduced I-function of two variables [5] which is a natural generalization of the $\mathrm{H}$-function of two variables introduced earlier by Mittal and Gupta [6] and discussed some of its important properties.

In the present paper, we aim to develop I-function of " $r$ " variables which may be regarded as the natural generalization of the H-function of " $r$ " variables introduced earlier by Srivastava and Panda [7]. We also discussed some of the important properties.

The remainder of this paper is organized as follows.
In Section 2, we have defined the I-function of " $r$ " variables by means of multiple Mellin-Barnes type contour integrals. In Section 3, we have given the convergence conditions for this function. In Section 4, we obtained the series representation and behaviour of the function for small values of the variables. In Section 5, we have mentioned special cases of our function giving relations with other functions available in the literature. Finally, in Section 6, we have mentioned a few important properties.

\section{The I-Function of Several Variables}

The generalized Fox H-function, namely, I-function of " $r$ " variables, is defined and represented in the following manner:

$$
\begin{aligned}
\mathrm{I}\left[z_{1}, \ldots, z_{r}\right] & =\mathrm{I}_{p, q: p_{1}, q_{1}, \ldots ; p_{r}, q_{r}}^{0, n: m_{1}, \ldots ; m_{r}, n_{r}}\left[\begin{array}{c|c}
z_{1} & \left(a_{j} ; \alpha_{j}^{(1)}, \ldots, \alpha_{j}^{(r)} ; A_{j}\right)_{1, p}:\left(c_{j}^{(1)}, \gamma_{j}^{(1)} ; C_{j}^{(1)}\right)_{1, p_{1}} ; \ldots ;\left(c_{j}^{(r)}, \gamma_{j}^{(r)} ; C_{j}^{(r)}\right)_{1, p_{r}} \\
\vdots & \left(b_{j} ; \beta_{j}^{(1)}, \ldots, \beta_{j}^{(r)} ; B_{j}\right)_{1, q}:\left(d_{j}^{(1)}, \delta_{j}^{(1)} ; D_{j}^{(1)}\right)_{1, q_{1}} ; \ldots ;\left(d_{j}^{(r)}, \delta_{j}^{(r)} ; D_{j}^{(r)}\right)_{1, q_{r}}
\end{array}\right] \\
& =\frac{1}{(2 \pi i)^{r}} \int_{\mathscr{L}_{1}} \cdots \int_{\mathscr{L}_{r}} \phi\left(s_{1}, \ldots, s_{r}\right) \theta_{1}\left(s_{1}\right) \cdots \theta_{r}\left(s_{r}\right) z_{1}^{s_{1}} \cdots z_{r}^{s_{r}} d s_{1} \ldots d s_{r},
\end{aligned}
$$


where $\phi\left(s_{1}, \ldots, s_{r}\right), \theta_{i}\left(s_{i}\right), i=1, \ldots, r$ are given by

$$
\begin{aligned}
\phi\left(s_{1}, \ldots, s_{r}\right)= & \prod_{j=1}^{n} \Gamma^{A_{j}}\left(1-a_{j}+\sum_{i=1}^{r} \alpha_{j}^{(i)} s_{i}\right) \\
& \times\left(\prod_{j=n+1}^{p} \Gamma^{A_{j}}\left(a_{j}-\sum_{i=1}^{r} \alpha_{j}^{(i)} s_{i}\right)\right. \\
\theta_{i}\left(s_{i}\right)= & \left(\prod_{j=1}^{n_{i}} \Gamma^{C_{j}^{(i)}}\left(1-c_{j}^{(i)}+\gamma_{j}^{(i)} s_{i}\right)\right. \\
& \left.\times \prod_{j=1}^{q} \Gamma^{m_{j}}\left(1-b_{j}+\sum_{i=1}^{r} \beta_{j}^{(i)} s_{i}\right)\right)^{-1}, \\
& \left.\times\left(\prod_{j=n_{i}+1}^{p_{i}^{(i)}}-\delta_{j}^{(i)} s_{i}\right)\right) \\
& \times \prod_{j=m_{i}+1}^{C_{i}^{(i)}}\left(c_{j}^{(i)}-\gamma_{j}^{(i)} s_{i}\right)
\end{aligned}
$$

where $i=1, \ldots, r$.

Also,

(i) $z_{i} \neq 0$, for $i=1, \ldots, r$;

(ii) $i=\sqrt{-1}$;

(iii) an empty product is interpreted as unity;

(iv) the parameters $m_{j}, n_{j}, p_{j}, q_{j}(j=1, \ldots, r), n, p$, and $q$ are nonnegative integers such that $0 \leq n \leq p, q \geq 0$, $0 \leq n_{j} \leq p_{j}$, and $0 \leq m_{j} \leq q_{j}(j=1, \ldots, r)$ (not all zero simulataneously);

(v) $\alpha_{j}^{(i)}(j=1, \ldots, p, i=1, \ldots, r), \beta_{j}^{(i)}(j=1, \ldots, q, i=$ $1, \ldots, r), \gamma_{j}^{(i)}\left(j=1, \ldots, p_{i}, i=1, \ldots, r\right)$, and $\delta_{j}^{(i)}(j=$ $\left.1, \ldots, q_{i}, i=1, \ldots, r\right)$ are assumed to be positive quantities for standardisation purpose. However, the definition of I-function of " $r$ " variables will have a meaning even if some of the quantities are zero or negative numbers. For these, we may obtain corresponding transformation formulas which will be given in a later section;

(vi) $a_{j}(j=1, \ldots, p), b_{j}(j=1, \ldots, q), c_{j}^{(i)}\left(j=1, \ldots, p_{i}\right.$, $i=1, \ldots, r)$, and $d_{j}^{(i)}\left(j=1, \ldots, q_{i}, i=1, \ldots, r\right)$ are complex numbers;

(vii) the exponents $A_{j}(j=1, \ldots, p), B_{j}(j=1, \ldots, q)$, $C_{j}^{(i)}\left(j=1, \ldots, p_{i}, i=1, \ldots, r\right)$, and $D_{j}^{(i)}(j=$ $\left.1, \ldots, q_{i}, i=1, \ldots, r\right)$ of various gamma functions involved in (2) and (3) may take noninteger values;

(viii) the contour $\mathscr{L}_{i}$ in the complex $s_{i}$-plane is of MellinBarnes type which runs from $c-i \infty$ to $c+i \infty$, ( $c$ real) with indentation, if necessary, in such a manner that all singularities of $\Gamma^{D_{j}^{(i)}}\left(d_{j}^{(i)}-\delta_{j}^{(i)} s_{i}\right), j=1, \ldots, m_{i}$ lie to the right and $\Gamma^{C_{j}^{(i)}}\left(1-c_{j}^{(i)}+\gamma_{j}^{(i)} s_{i}\right), j=1, \ldots, n_{i}$ are to the left of $\mathscr{L}_{i}$.

Following the results of Braaksma [8] the I-function of " $r$ " variables is analytic if

$$
\begin{array}{r}
\mu_{i}=\sum_{j=1}^{p} A_{j} \alpha_{j}^{(i)}-\sum_{j=1}^{q} B_{j} \beta_{j}^{(i)}+\sum_{j=1}^{p_{i}} C_{j}^{(i)} \gamma_{j}^{(i)} \\
-\sum_{j=1}^{q_{i}} D_{j}^{(i)} \delta_{j}^{(i)} \leq 0, \quad i=1, \ldots, r .
\end{array}
$$

\section{Convergence Conditions}

Integral (1) converges absolutely if

$$
\left|\arg \left(z_{k}\right)\right|<\frac{1}{2} \Delta_{k} \pi, \quad k=1, \ldots, r,
$$

where

$$
\begin{aligned}
\Delta_{k}=[ & -\sum_{j=n+1}^{p} A_{j} \alpha_{j}^{(k)}-\sum_{j=1}^{q} B_{j} \beta_{j}^{(k)} \\
& +\sum_{1}^{m_{k}} D_{j}^{(k)} \delta_{j}^{(k)}-\sum_{m_{k}+1}^{q_{k}} D_{j}^{(k)} \delta_{j}^{(k)} \\
& \left.+\sum_{j=1}^{n_{k}} C_{j}^{(k)} \gamma_{j}^{(k)}-\sum_{j=n_{k}+1}^{p_{k}} C_{j}^{(k)} \gamma_{j}^{(k)}\right]>0,
\end{aligned}
$$

and if $\left|\arg \left(z_{k}\right)\right|=(1 / 2) \Delta_{k} \pi$ and $\Delta_{k} \geq 0, k=1, \ldots, r$, then integral (1) converges absolutely under the following conditions:

(i) $\mu_{k}=0, \Omega_{k}<-1$, where $\mu_{k}$ is given by (4) and

$$
\begin{aligned}
& \Omega_{k}= \sum_{j=1}^{p}\left[\frac{1}{2}-\mathfrak{R}\left(a_{j}\right)\right] A_{j} \\
&-\sum_{j=1}^{q}\left[\frac{1}{2}-\mathfrak{R}\left(b_{j}\right)\right] B_{j} \\
&+\sum_{j=1}^{p_{k}}\left[\frac{1}{2}-\mathfrak{R}\left(c_{j}^{(k)}\right)\right] C_{j}^{(k)} \\
&-\sum_{j=1}^{q_{k}}\left[\frac{1}{2}-\mathfrak{R}\left(d_{j}^{(k)}\right)\right] D_{j}^{(k)}, \\
& k=1, \ldots, r ;
\end{aligned}
$$

(ii) $\mu_{k} \neq 0$ with $s_{k}=\sigma_{k}+i t_{k},\left(\sigma_{k}\right.$ and $t_{k}$ are real, $k=$ $1, \ldots, r)$, and $\sigma_{k}$ are chosen so that for $\left|t_{k}\right| \rightarrow \infty$ we have $\Omega_{k}+\sigma_{k} \mu_{k}<-1$.

Outline of the Proof. The convergence of integral (1) depends on the asymptotic behaviour of the functions $\phi\left(s_{1}, \ldots, s_{r}\right)$, $\theta_{i}\left(s_{i}\right), i=1, \ldots, r$ defined by (2) and (3), respectively. Such 
asymptotic behaviour is based on the following relation for the gamma function $\Gamma(z), z=x+i y, x, y \in \mathbb{R}[9]$ :

$$
|\Gamma(x+i y)| \sim \sqrt{2 \pi}|y|^{x-1 / 2} \exp \left(-\frac{1}{2} \pi|y|\right), \quad|y| \rightarrow \infty .
$$

Along the contour $\mathscr{L}_{k}$, if we put $s_{k}=\sigma_{k}+i t_{k}$ and take the limit as $\left|t_{k}\right| \rightarrow \infty$ for $k=1, \ldots, r$, we obtain by virtue of (8) that

$$
\begin{aligned}
& \left|\Gamma^{A_{j}}\left(1-a_{j}+\sum_{k=1}^{r} \alpha_{j}^{(k)} s_{k}\right)\right| \\
& \leq(2 \pi)^{A_{j} / 2}\left(\alpha_{j}^{(k)}\left|t_{k}\right|\right)^{\left[1 / 2-\Re\left(a_{j}\right)+\alpha_{j}^{(k)} \sigma_{k}\right] A_{j}} \\
& \quad \times \exp \left[-\frac{\pi}{2}\left(\alpha_{j}^{(k)}\left|t_{k}\right|+\left|\mathfrak{J}\left(a_{j}\right)\right|\right) A_{j}\right], \\
& \prod_{j=1}^{n}\left|\Gamma^{A_{j}}\left(1-a_{j}+\sum_{k=1}^{r} \alpha_{j}^{(k)} s_{k}\right)\right| \\
& \leq(2 \pi)^{\sum_{j=1}^{n}\left(A_{j} / 2\right)} \prod_{j=1}^{n}\left(\alpha_{j}^{(k)}\left|t_{k}\right|\right)^{\left[1 / 2-\Re\left(a_{j}\right)+\alpha_{j}^{(k)} \sigma_{k}\right] A_{j}} \\
& \quad \times \exp \left[-\frac{\Pi}{2} \sum_{j=1}^{n}\left(\alpha_{j}^{(k)}\left|t_{k}\right|+\left|\mathfrak{\Im}\left(a_{j}\right)\right|\right) A_{j}\right] .
\end{aligned}
$$

Similarly, we have

$$
\begin{aligned}
& \prod_{j=n+1}^{p}\left|\Gamma^{A_{j}}\left(a_{j}-\sum_{k=1}^{r} \alpha_{j}^{(k)} s_{k}\right)\right| \\
& \geq(2 \pi)^{\sum_{j=n+1}^{k}\left(A_{j} / 2\right)} \prod_{j=n+1}^{k}\left(\alpha_{j}^{(k)}\left|t_{k}\right|\right)^{\left[\Re\left(a_{j}\right)-\alpha_{j}^{(k)} \sigma_{k}-1 / 2\right] A_{j}} \\
& \quad \times \exp \left[-\frac{\pi}{2} \prod_{j=n+1}^{p}\left(\alpha_{j}^{(k)}\left|t_{k}\right|+\left|\mathfrak{J}\left(a_{j}\right)\right|\right) A_{j}\right] \\
& \prod_{j=1}^{q}\left|\Gamma^{B_{j}}\left(1-b_{j}+\sum_{k=1}^{r} \beta_{j}^{(k)} s_{k}\right)\right| \\
& \geq \quad(2 \pi)^{\sum_{j=1}^{q}\left(B_{j} / 2\right)} \prod_{j=1}^{q}\left(\beta_{j}^{(k)}\left|t_{k}\right|\right)^{\left[1 / 2-\Re\left(b_{j}\right)+\beta_{j}^{(k)} \sigma_{k}\right] B_{j}} \\
& \quad \times \exp \left[-\frac{\prod}{2} \prod_{j=1}^{q}\left(\beta_{j}^{(k)}\left|t_{k}\right|+\left|\Im\left(a_{j}\right)\right|\right) B_{j}\right] \\
& \prod_{j=1}^{n_{k}}\left|\Gamma_{j}^{(k)}\left(1-c_{j}^{(k)}+\gamma_{j}^{(k)} s_{k}\right)\right| \\
& \leq(2 \pi)^{\sum_{j=1}^{n_{k}}\left(C_{j}^{(k)} / 2\right)} \prod_{j=1}^{n_{k}}\left(\gamma_{j}^{(k)}\left|t_{k}\right|\right)^{\left[1 / 2-\Re\left(c_{j}^{(k)}\right)+\gamma_{j}^{(k)} \sigma_{k}\right] C_{j}^{(k)}} \\
& \quad \times \exp \left[-\frac{\prod}{2} \prod_{j=1}^{n_{k}}\left(\gamma_{j}^{(k)}\left|t_{k}\right|+\left|\mathfrak{\Im}\left(c_{j}^{(k)}\right)\right|\right) C_{j}^{(k)}\right]
\end{aligned}
$$

$$
\begin{aligned}
& \prod_{j=n_{k}+1}^{p_{k}}\left|\Gamma_{C_{j}^{(k)}}\left(c_{j}^{(k)}-\gamma_{j}^{(k)} s_{k}\right)\right| \\
& \geq(2 \pi)^{\sum_{j=n_{k}+1}^{p_{k}}\left(C_{j}^{(k)} / 2\right)} \prod_{j=n_{k}+1}^{p_{k}}\left(\gamma_{j}^{(k)}\left|t_{k}\right|\right)^{\left[\Re\left(c_{j}^{(k)}\right)-\gamma_{j}^{(k)} \sigma_{k}-1 / 2\right] C_{j}^{(k)}} \\
& \times \exp \left[-\frac{\pi}{2} \prod_{j=n_{k}+1}^{p_{k}}\left(\gamma_{j}^{(k)}\left|t_{k}\right|+\left|\mathfrak{J}\left(c_{j}^{(k)}\right)\right|\right) C_{j}^{(k)}\right] \text {, } \\
& \prod_{j=1}^{m_{k}}\left|\Gamma^{D_{j}^{(k)}}\left(d_{j}^{(k)}-\delta_{j}^{(k)} s_{k}\right)\right| \\
& \leq(2 \pi)^{\sum_{j=1}^{m_{k}}\left(D_{j}^{(k)} / 2\right)} \prod_{j=1}^{m_{k}}\left(\delta_{j}^{(k)}\left|t_{k}\right|\right)^{\left[\Re\left(d_{j}^{(k)}\right)+\delta_{j}^{(k)} \sigma_{k}-1 / 2\right] D_{j}^{(k)}} \\
& \times \exp \left[-\frac{\pi}{2} \prod_{j=1}^{m_{k}}\left(\delta_{j}^{(k)}\left|t_{k}\right|+\left|\mathfrak{\Im}\left(d_{j}^{(k)}\right)\right|\right) D_{j}^{(k)}\right], \\
& \prod_{j=m_{k}+1}^{q_{k}}\left|\Gamma^{D_{j}^{(k)}}\left(1-d_{j}^{(k)}+\delta_{j}^{(k)} s_{k}\right)\right| \\
& \leq(2 \pi)^{\sum_{j=m_{k}+1}^{q_{k}}\left(D_{j}^{(k)} / 2\right)} \prod_{j=m_{k}+1}^{q_{k}}\left(\delta_{j}^{(k)}\left|t_{k}\right|\right)^{\left[1 / 2-\Re\left(d_{j}^{(k)}\right)+\delta_{j}^{(k)} \sigma_{k}\right] D_{j}^{(k)}} \\
& \times \exp \left[-\frac{\pi}{2} \prod_{j=m_{k}+1}^{q_{k}}\left(\delta_{j}^{(k)}\left|t_{k}\right|+\left|\mathfrak{J}\left(d_{j}^{(k)}\right)\right|\right) D_{j}^{(k)}\right] \text {. }
\end{aligned}
$$

Also,

$$
\begin{aligned}
z_{k}^{s_{k}} & =\exp \left[\left(\sigma_{k}+i t_{k}\right)\left(\log \left|z_{k}\right|+i \arg \left(z_{k}\right)\right)\right] \\
& =\exp \left[\sigma_{k} \log \left|z_{k}\right|-t_{k} \arg \left(z_{k}\right)\right] \\
& =\left|z_{k}\right|^{\sigma_{k}} \exp \left[-t_{k} \arg \left(z_{k}\right)\right] .
\end{aligned}
$$

Hence, substituting (10)-(11) in (1) and using (12) we have, after much simplification,

$$
\begin{aligned}
& \left|\phi\left(s_{1}, \ldots, s_{r}\right) \theta_{k}\left(s_{k}\right) z_{k}^{s_{k}}\right| \\
& \quad \sim C_{k}\left|t_{k}\right|^{\Omega_{k}+\mu_{k} \sigma_{k}} \exp \left[-t_{k} \arg \left(z_{k}\right)-\frac{\pi}{2}\left|t_{k}\right| \Delta_{k}\right],
\end{aligned}
$$

where $C_{k}$ is independent of $t_{k}$ and $\Delta_{k}, \mu_{k}$, and $\Omega_{k}$ are given by (6), (7), and (8), respectively, for each $k=1,2, \ldots, r$.

Hence, the result follows. 
Remark 1. If $D_{j}^{(i)}=1\left(j=1, \ldots, m_{i}, i=1, \ldots, r\right)$ in (1), then the function will be denoted by

$$
\begin{aligned}
\overline{\mathrm{I}}\left[\begin{array}{c}
z_{1} \\
\vdots \\
z_{r}
\end{array}\right]= & \mathrm{I}_{p, q: p_{1}, q_{1}, \ldots ; p_{r}, q_{r}}^{0, n: m_{1}, n_{1} ; \ldots ; m_{r}, n_{r}} \\
& \left.\left.\times\left[\begin{array}{c}
z_{1} \\
\vdots \\
z_{r}
\end{array} \mid\left(a_{j} ; \alpha_{j}^{(1)}, \ldots, \alpha_{j}^{(r)} ; A_{j}\right)_{1, p}:\left(\beta_{j}^{(1)}, \ldots, \beta_{j}^{(r)} ; B_{j}\right)_{1, q}:\left(d_{j}^{(1)}, \delta_{j}^{(1)} ; 1\right)_{1, m_{1}}^{(1)}, C_{j}^{(1)}\right)_{1, p_{1}} ; \ldots ;\left(d_{j}^{(1)}, \delta_{j}^{(1)} ; D_{j}^{(1)}\right)_{m_{1}+1, q_{1}} ; \ldots ;\left(\gamma_{j}^{(r)} ; C_{j}^{(r)}\right)_{1, p_{r}}, \delta_{j}^{(r)} ; 1\right)_{1, m_{r}},\left(d_{j}^{(r)}, \delta_{j}^{(r)} ; D_{j}^{(r)}\right)_{m_{r}+1, q_{r}}\right] \\
= & \frac{1}{(2 \pi i)^{r}} \int_{\mathscr{L}_{1}} \ldots \int_{\mathscr{L}_{r}} \phi\left(s_{1}, \ldots, s_{r}\right) \overline{\theta_{1}}\left(s_{1}\right) \ldots \overline{\theta_{r}}\left(s_{r}\right) z_{1}^{s_{1}} \cdots z_{r}^{s_{r}} d s_{1} \ldots d s_{r},
\end{aligned}
$$

where

$$
\begin{aligned}
\overline{\theta_{i}}\left(s_{i}\right)= & \prod_{j=1}^{n_{i}} \Gamma^{C_{j}^{(i)}}\left(1-c_{j}^{(i)}+\gamma_{j}^{(i)} s_{i}\right) \prod_{j=1}^{m_{i}} \Gamma\left(d_{j}^{(i)}-\delta_{j}^{(i)} s_{i}\right) \\
& \times\left(\prod_{j=n_{i}+1}^{p_{i}} \Gamma^{C_{j}^{(i)}}\left(c_{j}^{(i)}-\gamma_{j}^{(i)} s_{i}\right) \prod_{j=m_{i}+1}^{q_{i}} \Gamma^{D_{j}^{(i)}}\left(1-d_{j}^{(i)}+\delta_{j}^{(i)} s_{i}\right)\right)^{-1},
\end{aligned}
$$

where $i=1, \ldots, r$.
Remark 2. If $C_{j}^{(i)}=1\left(j=1, \ldots, n_{i}\right), D_{j}^{(i)}=1\left(j=1, \ldots, m_{i}\right)$, where $i=1, \ldots, r$ and if $n=0$ in (1), then the corresponding function will be denoted by

$$
\begin{aligned}
\overline{\mathrm{I}}_{1}\left[\begin{array}{c}
z_{1} \\
\vdots \\
z_{r}
\end{array}\right]= & \mathrm{I}_{p, q: p_{1}, q_{1} ; \ldots ; p_{r}, q_{r}}^{0,0 m_{1}, n_{1} ; \ldots ; m_{r}, n_{r}} \\
& \left.\times\left[\begin{array}{c}
z_{1} \\
\vdots \\
z_{r}
\end{array}\left(a_{j} ; \alpha_{j}^{(1)}, \ldots, \alpha_{j}^{(r)} ; A_{j}\right)_{1, p}:\left(c_{j}^{(1)}, \gamma_{j}^{(1)} ; 1\right)_{1, n_{1}},\left(c_{j}^{(1)}, \gamma_{j}^{(1)} ; C_{j}^{(1)}\right)_{n_{1}+1, p_{1}} ; \ldots ;\left(c_{j}^{(r)}, \gamma_{j}^{(r)} ; 1\right)_{1, n_{r}},\left(B_{j}\right)_{1, q}:\left(d_{j}^{(1)}, \delta_{j}^{(1)} ; 1\right)_{1, m_{1}}^{(r)} ; C_{j}^{(r)}\right)_{n_{r}+1, p_{r}},\left(d_{j}^{(1)}, \delta_{j}^{(1)} ; D_{j}^{(1)}\right)_{m_{1}+1, q_{1}} ; \ldots ;\left(d_{j}^{(r)}, \delta_{j}^{(r)} ; 1\right)_{1, m_{r}},\left(d_{j}^{(r)}, \delta_{j}^{(r)} ; D_{j}^{(r)}\right)_{m_{r}+1, q_{r}}\right] \\
= & \frac{1}{(2 \pi i)^{r}} \int_{\mathscr{L}_{1}} \cdots \int_{\mathscr{L}_{r}} \phi_{1}\left(s_{1}, \ldots, s_{r}\right) \overline{\theta_{1}}\left(s_{1}\right) \cdots \overline{\theta_{r}}\left(s_{r}\right) z_{1}^{s_{1}} \cdots z_{r}^{s_{r}} d s_{1} \cdots d s_{r},
\end{aligned}
$$

where

$$
\begin{aligned}
& \phi_{1}\left(s_{1}, \ldots, s_{r}\right) \\
& =\frac{1}{\prod_{j=1}^{p} \Gamma^{A_{j}}\left(a_{j}-\sum_{i=1}^{r} \alpha_{j}^{(i)} s_{i}\right) \prod_{j=1}^{q} \Gamma^{B_{j}}\left(1-b_{j}+\sum_{i=1}^{r} \beta_{j}^{(i)} s_{i}\right)}, \\
& \overline{\theta_{i}}\left(s_{i}\right)=\frac{\prod_{j=1}^{n_{i}} \Gamma\left(1-c_{j}^{(i)}+\gamma_{j}^{(i)} s_{i}\right) \prod_{j=1}^{m_{i}} \Gamma\left(d_{j}^{(i)}-\delta_{j}^{(i)} s_{i}\right)}{\prod_{j=n_{i}+1}^{p_{i}} \Gamma^{C_{j}^{(i)}}\left(c_{j}^{(i)}-\gamma_{j}^{(i)} s_{i}\right) \prod_{j=m_{i}+1}^{q_{i}} \Gamma^{D_{j}^{(i)}}\left(1-d_{j}^{(i)}+\delta_{j}^{(i)} s_{i}\right)} \\
& \forall i=1, \ldots, r .
\end{aligned}
$$

\section{Series Representation}

if

(i) $z_{i} \neq 0,(i=1, \ldots, r)$ and $\mu_{i}<0$, where $\mu_{i}$ is given by (4); (ii) $\delta_{h_{i}}^{(i)}\left(d_{j}^{(i)}+k_{i}\right) \neq \delta_{j}^{(i)}\left(d_{h_{i}}^{(i)}+\eta_{i}\right)$ for $j \neq h_{i}, j, h_{i}=1, \ldots, m_{i}$, $(i=1, \ldots, r), k_{i}, \eta_{i}=0,1,2, \ldots(i=1, \ldots, r)$,

then

$$
\begin{aligned}
\overline{\mathrm{I}}\left[\begin{array}{c}
z_{1} \\
\vdots \\
z_{r}
\end{array}\right]= & \sum_{h_{1}=1}^{m_{1}} \cdots \sum_{h_{r}=1}^{m_{r}} \sum_{k_{1}=1}^{\infty} \ldots \sum_{k_{r}=1}^{\infty} \\
\times & {\left[\phi_{1}\left(\frac{d h_{1}^{(1)}+k_{1}}{\delta h_{1}^{(1)}}, \ldots, \frac{d h_{r}^{(r)}+k_{r}}{\delta h_{r}^{(r)}}\right)\right.} \\
& \left.\times \prod_{i=1}^{r} \frac{(-1)^{k_{i}}}{\delta h_{i}^{(i)} k_{i} !} z_{i}^{\left(d h_{i}+k_{i}\right) / \delta h_{i}}\right]_{j \neq h_{i}} .
\end{aligned}
$$

This result can be proved on computing the residues at the 
poles as follows:

$$
\begin{aligned}
s_{r}= & \frac{d h_{i}^{(i)}+k_{i}}{\delta h_{i}^{(i)}}, \\
& \left(h_{i}=1, \ldots, m_{i}, k_{i}=0,1,2, \ldots\right) \text { for } i=1, \ldots, r .
\end{aligned}
$$

The behaviour of the function $\overline{\mathrm{I}}\left[\begin{array}{c}z_{1} \\ \vdots \\ z_{r}\end{array}\right]$ is given by

$$
\overline{\mathrm{I}}\left[\begin{array}{c}
z_{1} \\
\vdots \\
z_{r}
\end{array}\right]=O\left(\prod_{j=1}^{r}\left|z_{i}\right|^{\phi_{j}}\right), \quad \max \left\{\left|z_{1}\right|, \ldots,\left|z_{r}\right|\right\} \rightarrow 0,
$$

where

$$
\phi_{j}=\min _{1 \leq j \leq m_{i}}\left[\operatorname{Re}\left(\frac{d_{j}^{(i)}}{\delta_{j}^{(i)}}\right)\right], \quad(i=1, \ldots, r) .
$$

On the other hand, when $\left|z_{i}\right| \rightarrow \infty(i=1, \ldots, r)$, the associated function $\overline{\mathrm{I}}_{1}\left[\begin{array}{c}z_{1} \\ \vdots \\ z_{r}\end{array}\right]$ given by (16) has the behaviour $\overline{\mathrm{I}}_{1}\left[\begin{array}{c}z_{1} \\ \vdots \\ z_{r}\end{array}\right]=\mathrm{O}\left(\prod_{j=1}^{r}\left|z_{j}\right|^{\phi_{j}}\right), \quad \min \left\{\left|z_{1}\right|, \ldots,\left|z_{r}\right|\right\} \rightarrow 0$, where

$$
\phi_{j}=\max _{1 \leq j \leq n_{i}}\left[\operatorname{Re}\left(\frac{1-c_{j}^{(i)}}{\gamma_{j}^{(i)}}\right)\right], \quad(i=1, \ldots, r) .
$$

\section{Elementary Special Cases}

In this section, we mention some interesting and useful special cases of the I-function of " $r$ " variables.

(i) If all the exponents $A_{j}(j=1, \ldots, p), B_{j}(j=$ $1, \ldots, q), C_{j}^{(i)}\left(j=1, \ldots, p_{i}, i=1, \ldots, r\right)$, and $D_{j}^{(i)}\left(j=1, \ldots, q_{i}, i=1, \ldots, r\right)$ in (1) are equal to unity, we obtain $\mathrm{H}$-function of " $r$ " variables defined by Srivastava and Panda [7].

(ii) When $p=q=n=0$, (1) degenerates into the product of $r$ mutually independent I- functions of one variable introduced by Rathie [1].

(iii) When $p=q=n=0$ and $r=1$, (1) reduces to the I-function defined by Rathie [1].

(iv) When $n=p, m_{i}=1, n_{i}=p_{i}, i=1, \ldots, r$, and $A_{j}=$ $B_{j}=C_{j}=D_{j}=1$ and $\left(d_{j}^{(i)}, \delta_{j}^{(i)} ; D_{j}^{(i)}\right)$ is replaced by $(0,1,1),\left(d_{j}^{(i)}, \delta_{j}^{(i)} ; D_{j}^{(i)}\right),(1)$ reduces to the generalized Lauricella function [10].

$$
\begin{aligned}
& \mathrm{I}_{p, q: p: 1, p_{1}, q_{1}+\ldots, 1, \ldots, p_{r}, q_{r}+1}\left[\begin{array}{c|l}
z_{1} & \left(a_{j} ; \alpha_{j}^{(1)}, \ldots, \alpha_{j}^{(r)} ; 1\right)_{1, p}:\left(c_{j}^{(1)}, \gamma_{j}^{(1)} ; 1\right)_{1, p_{1}} ; \ldots ;\left(c_{j}^{(r)}, \gamma_{j}^{(r)} ; 1\right)_{1, p_{r}} \\
\vdots & \left(b_{j} ; \beta_{j}^{(1)}, \beta_{j}^{(1)}, \ldots, \beta_{j}^{(r)} ; 1\right)_{1, q}:(0,1 ; 1),\left(d_{j}^{(1)}, \delta_{j}^{(1)} ; 1\right)_{1, q_{1}} ; \ldots ;(0,1 ; 1),\left(d_{j}^{(r)}, \delta_{j}^{(r)} ; 1\right)_{1, q_{r}}
\end{array}\right] \\
& =\frac{\prod_{j=1}^{p} \Gamma\left(1-a_{j}\right) \prod_{j=1}^{p_{1}} \Gamma\left(1-c_{j}^{(1)}\right) \cdots \prod_{j=1}^{p_{r}} \Gamma\left(1-c_{j}^{(r)}\right)}{\prod_{j=1}^{q} \Gamma\left(1-b_{j}\right) \prod_{j=1}^{q_{1}} \Gamma\left(1-d_{j}^{(1)}\right) \cdots \prod_{j=1}^{q_{r}} \Gamma\left(1-d_{j}^{(r)}\right)} \\
& \quad \times F_{q: q_{1} ; \ldots ; q_{r}}^{p: p_{1} ; \ldots ; p_{r}}\left[\begin{array}{c|c}
-z_{1} & \left(1-a_{j} ; \alpha_{j}^{(1)}, \ldots, \alpha_{j}^{(r)}\right)_{1, p}:\left(1-c_{j}^{(1)}, \gamma_{j}^{(1)}\right)_{1, p_{1}} ; \ldots ;\left(1-c_{j}^{(r))}, \gamma_{j}^{(r)}\right)_{1, p_{r}} \\
\vdots & \left(1-b_{j} ; \beta_{j}^{(1)}, \ldots, \beta_{j}^{(r)}\right)_{1, q}:\left(1-d_{j}^{(1)}, \delta_{j}^{(1)}\right)_{1, q_{1}} ; \ldots ;\left(1-d_{j}^{(r)}, \delta_{j}^{(r)}\right)_{1, q_{r}}
\end{array}\right] .
\end{aligned}
$$

$$
={ }_{p_{1}} \Psi_{q_{1}}\left[\begin{array}{l}
\left(c_{j}^{(1)}, \gamma_{j}^{(1)}\right)_{1, p_{1}} ; z_{1} \\
\left(d_{j}^{(1)}, \delta_{j}^{(1)}\right)_{1, q_{1}}
\end{array}\right] \times \cdots \times{ }_{p_{r}} \Psi_{q_{r}}\left[\begin{array}{l}
\left(c_{j}^{(r)}, \gamma_{j}^{(r)}\right)_{1, p_{r}} ; z_{r} \\
\left(d_{j}^{(r)}, \delta_{j}^{(r)}\right)_{1, q_{r}}
\end{array}\right] \text {, }
$$


where the functions $p_{i} \Psi_{q_{i}}, i=1, \ldots, r$ are Wright's generalized hypergeometric functions [11].

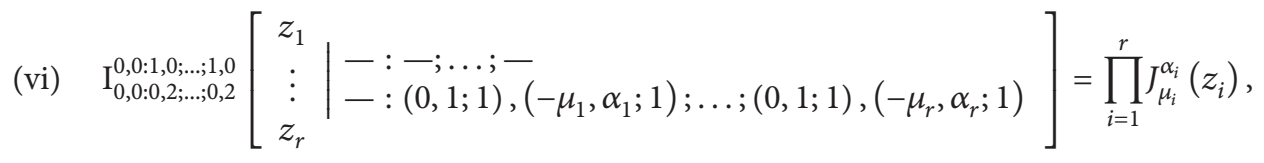

where the functions $J_{\mu_{i}}^{\alpha_{i}}\left(z_{i}\right)$ are Wright's generalized Bessel functions [12].

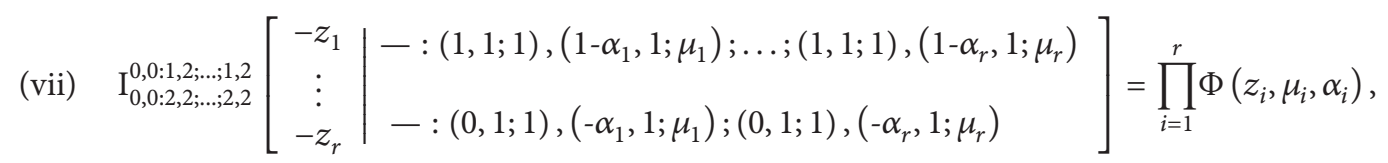

where $\Phi\left(z_{i}, \mu_{i}, \alpha_{i}\right), i=1, \ldots, r$ are the generalized Riemann zeta functions $[13$, page $27,1.11,(1)]$, which are the generaliza- tions of Hurwitz zeta functions and Riemann zeta functions $[13$, page $24,1.10,(1)$ and $1.12,(1)]$.

$$
\text { (viii) } \quad \mathrm{I}_{0,0: 2,2 ; \ldots ; 2,2}^{0,0: 1,2 ; .1,2}\left[\begin{array}{c|c}
-z_{1} & -:(1,1 ; 1),\left(1,1 ; \mu_{1}\right) ; \ldots ;(1,1 ; 1),\left(1,1 ; \mu_{r}\right) \\
\vdots & -:(0,1 ; 1),\left(0,1 ; \mu_{1}\right) ; \ldots ;(0,1 ; 1),\left(0,1 ; \mu_{r}\right)
\end{array}\right]=\prod_{i=1}^{r} F\left(z_{i}, \mu_{i}\right)
$$

where $F\left(z_{i}, \mu_{i}\right)$ are the polylogarithms of order $\mu_{i}$. For $\mu_{i}=2$, $i=1, \ldots, r$, the R.H.S. of (28) reduces to the product of Euler's dilogarithm [13, page 31, 1.11.1, equation (2)].

\section{Elementary Properties and Transformation Formulas}

The properties given below are immediate consequence of the definition (1) and hence they are given here without proof:

(i)

$$
\begin{array}{r}
\mathrm{I}_{p, q: p_{1}, q_{1}, \ldots ; p_{r}, q_{r}}^{0,0: m_{1}, n_{1} ; \ldots, m_{r}, n_{r}}\left[\begin{array}{c|c}
z_{1} & \left(a_{j} ; \alpha_{j}^{(1)}, \ldots, \alpha_{j}^{(r)} ; A_{j}\right)_{1, p}:\left(c_{j}^{(1)}, \gamma_{j}^{(1)} ; C_{j}^{(1)}\right)_{1, p_{1}} ; \ldots ;\left(c_{j}^{(r)}, \gamma_{j}^{(r)} ; C_{j}^{(r)}\right)_{1, p_{r}} \\
\vdots & \left(b_{j} ; \beta_{j}^{(1)}, \ldots, \beta_{j}^{(r)} B_{j}\right)_{1, q}:\left(d_{j}^{(1)}, \delta_{j}^{(1)} ; D_{j}^{(1)}\right)_{1, q_{1}} ; \ldots ;\left(d_{j}^{(r)}, \delta_{j}^{(r)} ; D_{j}^{(r)}\right)_{1, q_{r}}
\end{array}\right] \\
=\mathrm{I}_{q, p: q_{1}, p_{1} ; \ldots ; q_{r}, p_{r}}^{0,0: n_{1}, m_{1} ; \ldots, n_{r}, m_{r}}\left[\begin{array}{c}
z_{1}^{-1} \\
\vdots \\
z_{r}^{-1}
\end{array} \mid \begin{array}{l}
\left(1-b_{j} ; \beta_{j}^{(1)}, \ldots, \beta_{j}^{(r)} ; B_{j}\right)_{1, q}:\left(1-d_{j}^{(1)}, \delta_{j}^{(1)} ; D_{j}^{(1)}\right)_{1, q_{1}} ; \ldots ;\left(1-d_{j}^{(r)}, \delta_{j}^{(r)} ; D_{j}^{(r)}\right)_{1, q_{r}} \\
\left.z_{j}, \ldots, \alpha_{j}^{(r)} ; A_{j}\right)_{1, p}:\left(1-c_{j}^{(1)}, \gamma_{j}^{(1)} ; C_{j}^{(1)}\right)_{1, p_{1}} ;\left(1-c_{j}^{(r)}, \gamma_{j}^{(r)} ; C_{j}^{(r)}\right)_{1, p_{r}}
\end{array}\right],
\end{array}
$$

(ii)

$$
\begin{aligned}
& z_{1}^{k_{1}} \cdots z_{r}^{k_{r}} \mathrm{I}\left[z_{1} \cdots z_{r}\right] \\
& =\mathrm{I}_{p, q: p_{1}, q_{1} ; \ldots ; p_{r}, q_{r}}^{0, n: m_{1}, n_{1} ; \ldots ; m_{r}, n_{r}}\left[\begin{array}{c|c}
z_{1} & \left.\left(a_{j}+\sum_{i=1}^{r} k_{i} \alpha_{j}^{(i)} ; \alpha_{j}^{(1)}, \ldots, \alpha_{j}^{(r)} ; A_{j}\right)_{1, p}:\left(c_{j}^{(1)}+k_{1} \gamma_{j}^{(1)}, \gamma_{j}^{(1)} ; C_{j}^{(1)}\right)_{1, p_{1}} ; \ldots ;\left(c_{j}^{(r)}+k_{r} \gamma_{j}^{(r)}, \gamma_{j}^{(r)} ; C_{j}^{(r)}\right)_{1, p_{r}}\right] \\
z_{r} & \left.\left(b_{j}+\sum_{i=1}^{r} k_{i} \beta_{j}^{(i)} ; \beta_{j}^{(1)}, \ldots, \beta_{j}^{(r)} ; B_{j}\right)_{1, q}:\left(d_{j}^{(1)}+k_{1} \delta_{j}^{(1)}, \delta_{j}^{(1)} ; D_{j}^{(1)}\right)_{1, q_{1}} ; \ldots ;\left(d_{j}^{(r)}+k_{r} \delta_{j}^{(r)}, \delta_{j}^{(r)} ; D_{j}^{(r)}\right)_{1, q_{r}}\right],
\end{array}\right.
\end{aligned}
$$

for $k_{i}>0, i=1, \ldots r$ 
(iii) $\frac{1}{k_{1}} \cdots \frac{1}{k_{r}} \mathrm{I}\left[z_{1}, \ldots z_{r}\right]$

$$
=\mathrm{I}_{p, q: p_{1}, q_{1} ; \ldots ; p_{r}, q_{r}}^{0, n: q_{r}, q_{r} ;}\left[\begin{array}{c|c}
z_{1}^{k_{1}} & \left(a_{j} ; k_{1} \alpha_{j}^{(1)}, \ldots, k_{r} \alpha_{j}^{(r)} ; A_{j}\right)_{1, p}:\left(c_{j}^{(1)}, k_{1} \gamma_{j}^{(1)} ; C_{j}^{(1)}\right)_{1, p_{1}} ; \ldots ;\left(c_{j}^{(r)}, k_{r} \gamma_{j}^{(r)} ; C_{j}^{(r)}\right)_{1, p_{r}} \\
\vdots & \left(b_{j} ; k_{1} \beta_{j}^{(1)}, \ldots, k_{r} \beta_{j}^{(r)} ; B_{j}\right)_{1, p}:\left(d_{j}^{(1)}, k_{1} \delta_{j}^{(1)} ; D_{j}^{(1)}\right)_{1, q_{1}} ; \ldots ;\left(d_{j}^{(r)}, k_{r} \delta_{j}^{(r)} ; D_{j}^{(r)}\right)_{1, q_{r}}
\end{array}\right],
$$

where $k_{i}>0, i=1, \ldots, r$,

(iv) $\quad \mathrm{I}_{p, q: p_{1}, q_{1} ; \ldots, \ldots p_{r}, q_{r}}^{0, n: q_{r}, n_{r}}\left[\begin{array}{c|l}z_{1} & (a ; \alpha, 0, \ldots, 0 ; A),\left(a_{j} ; \alpha_{j}^{(1)}, \ldots, \alpha_{j}^{(r)} ; A_{j}\right)_{2, p}:\left(c_{j}^{(1)}, \gamma_{j}^{(1)} ; C_{j}^{(1)}\right)_{1, p_{1}} ; \ldots ;\left(c_{j}^{(r)}, \gamma_{j}^{(r)} ; C_{j}^{(r)}\right)_{1, p_{r}} \\ \vdots & \left(b_{j} ; \beta_{j}^{(1)}, \ldots, \beta_{j}^{(r)} ; B_{j}\right)_{1, q}:\left(d_{j}^{(1)}, \delta_{j}^{(1)} ; D_{j}^{(1)}\right)_{1, q_{1}}, \ldots,\left(d_{j}^{(r)}, \delta_{j}^{(r)} ; D_{j}^{(r)}\right)_{1, q_{r}}\end{array}\right]$

$=\mathrm{I}_{p, q: p_{1}+1, q_{1} ; \ldots ; p_{r}, q_{r}}^{0, n-1, n_{r}}\left[\begin{array}{c|l}z_{1} & \left(a_{j} ; \alpha_{j}^{(1)}, \ldots, \alpha_{j}^{(r)} ;\right)_{2, p}:(a, \alpha ; A),\left(c_{j}^{(1)}, \gamma_{j}^{(1)} ; C_{j}^{(1)}\right)_{1, p_{1}} ; \ldots ;\left(c_{j}^{(r)}, \gamma_{j}^{(r)} ; C_{j}^{(r)}\right)_{1, p_{r}} \\ \vdots & \left(b_{j} ; \beta_{j}^{(1)}, \ldots, \beta_{j}^{(r)} ; B_{j}\right)_{1, q}:\left(d_{j}^{(1)}, \delta_{j}^{(1)} ; D_{j}^{(1)}\right)_{1, q_{1}} ; \ldots ;\left(d_{j}^{(r)}, \delta_{j}^{(r)} ; D_{j}^{(r)}\right)_{1, q_{r}}\end{array}\right]$,

where $p \geq n \geq 1$,

(v)

$$
\begin{aligned}
& \mathrm{I}_{p, q: p_{1}, q_{1}, \ldots ; p_{r}, q_{r}}^{0, n m_{r}, n_{1} ; \ldots m_{r}, n_{r}}\left[\begin{array}{c|c}
z_{1} & \left(a_{j} ; \alpha_{j}^{(1)}, \ldots \alpha_{j}^{(r)} ; A_{j}\right)_{1, p-1},(a ; \alpha, 0, \ldots, 0 ; A):\left(c_{j}^{(1)}, \gamma_{j}^{(1)} ; C_{j}^{(1)}\right)_{1, p_{1}} ; \ldots ;\left(c_{j}^{(r)}, \gamma_{j}^{(r)} ; C_{j}^{(r)}\right)_{1, p_{r}} \\
\vdots & \left(b_{j} ; \beta_{j}^{(1)}, \ldots, \beta_{j}^{(r)} ; B_{j}\right)_{1, q}:\left(d_{j}^{(1)}, \delta_{j}^{(1)} ; D_{j}^{(1)}\right)_{1, q_{1}} ; \ldots ;\left(d_{j}^{(r)}, \delta_{j}^{(r)} ; D_{j}^{(r)}\right)_{1, q_{r}}
\end{array}\right] \\
& =\mathrm{I}_{p-1, q: m_{1}, 1, m_{1}, q_{1}, \ldots ; p_{r}, q_{r}}\left[\begin{array}{c|l}
z_{1} & \left(a_{j} ; \alpha_{j}^{(1)}, \ldots \alpha_{j}^{(r)} ; A_{j}\right)_{1, p-1}:\left(c_{j}^{(1)}, \gamma_{j}^{(1)} ; C_{j}^{(1)}\right)_{1, p_{1}},(a, \alpha ; A) ; \ldots ;\left(c_{j}^{(r)}, \gamma_{j}^{(r)} ; C_{j}^{(r)}\right)_{1, p_{r}} \\
\vdots & \left(b_{j} ; \beta_{j}^{(1)}, \ldots, \beta_{j}^{(r)} ; B_{j}\right)_{1, q}:\left(d_{j}^{(1)}, \delta_{j}^{(1)} ; D_{j}^{(1)}\right)_{1, q_{1}} ; \ldots ;\left(d_{j}^{(r)}, \delta_{j}^{(r)} ; D_{j}^{(r)}\right)_{1, q_{r}}
\end{array}\right],
\end{aligned}
$$

where $p-1 \geq n \geq 0$,

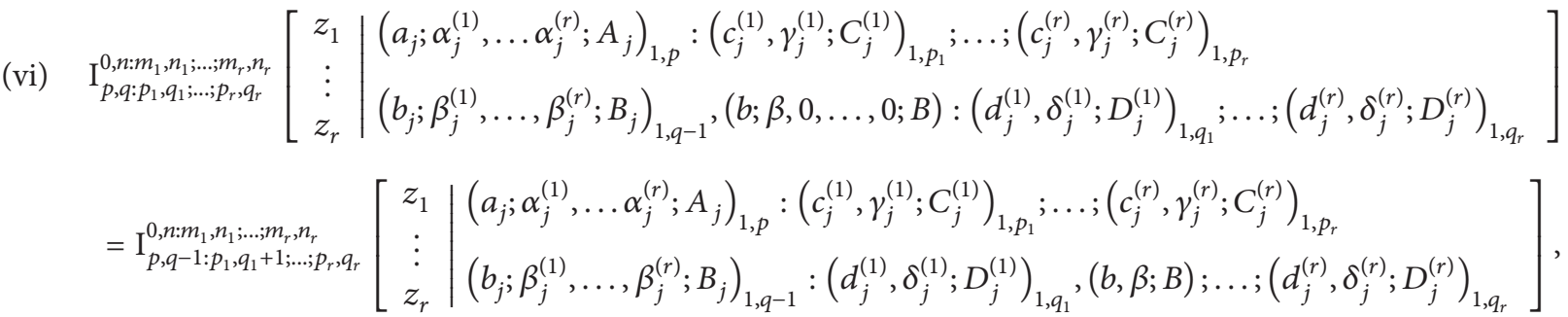

where $q-1 \geq 0$, 
8

International Journal of Engineering Mathematics

(vii)

$$
\begin{aligned}
& \mathrm{I}_{p, q: p_{1}, q_{1}, \ldots ; p_{r}, q_{r}}^{0, n: m_{1}, n_{1} ; \ldots m_{r}, n_{r}}\left[\begin{array}{c|l}
z_{1} & (a ; 0, \ldots, 0 ; A),\left(a_{j} ; \alpha_{j}^{(1)}, \ldots \alpha_{j}^{(r)} ; A_{j}\right)_{2, p}:\left(c_{j}^{(1)}, \gamma_{j}^{(1)} ; C_{j}^{(1)}\right)_{1, p_{1}} ; \ldots ;\left(c_{j}^{(r)}, \gamma_{j}^{(r)} ; C_{j}^{(r)}\right)_{1, p_{r}} \\
\vdots & \left(b_{j} ; \beta_{j}^{(1)}, \ldots, \beta_{j}^{(r)} ; B_{j}\right)_{1, q}:\left(d_{j}^{(1)}, \delta_{j}^{(1)} ; D_{j}^{(1)}\right)_{1, q_{1}} ; \ldots ;\left(d_{j}^{(r)}, \delta_{j}^{(r)} ; D_{j}^{(r)}\right)_{1, q_{r}}
\end{array}\right] \\
& =\Gamma^{A}(1-a) \times \mathrm{I}_{p-1, q: p_{1}, q_{1} ; \ldots ; p_{r}, q_{r}}^{0, n-1: n_{r}, \ldots ; n_{r}}\left[\begin{array}{c|l}
z_{1} & \left(a_{j} ; \alpha_{j}^{(1)}, \ldots \alpha_{j}^{(r)} ; A_{j}\right)_{2, p}:\left(c_{j}^{(1)}, \gamma_{j}^{(1)} ; C_{j}^{(1)}\right)_{1, p_{1}} ; \ldots ;\left(c_{j}^{(r)}, \gamma_{j}^{(r)} ; C_{j}^{(r)}\right)_{1, p_{r}} \\
\vdots & \left(b_{j} ; \beta_{j}^{(1)}, \ldots, \beta_{j}^{(r)} ; B_{j}\right)_{1, q}:\left(d_{j}^{(1)}, \delta_{j}^{(1)} ; D_{j}^{(1)}\right)_{1, q_{1}} ; \ldots ;\left(d_{j}^{(r)}, \delta_{j}^{(r)} ; D_{j}^{(r)}\right)_{1, q_{r}}
\end{array}\right],
\end{aligned}
$$

where $p \geq n \geq 1, \mathfrak{R}(1-a)>0$,

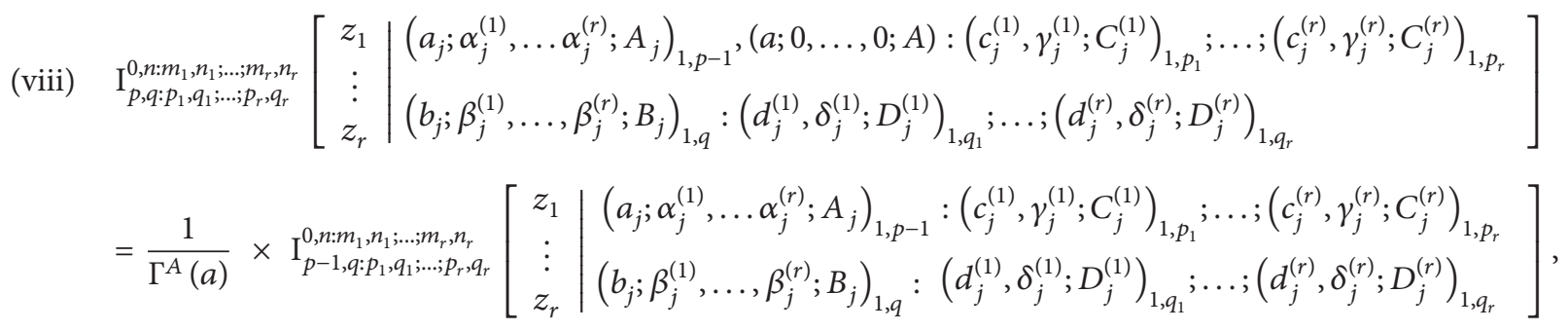

where $p-1 \geq n \geq 0, \mathfrak{R}(a)>0$,

(ix)

$$
\begin{aligned}
& \mathrm{I}_{p, q: p_{1}, q_{1} ; \ldots ; p_{r}, q_{r}}^{0, n: m_{1}, n_{1} ; \ldots m_{r_{2}}}\left[\begin{array}{c|c}
z_{1} & \left(a_{j} ; \alpha_{j}^{(1)}, \ldots \alpha_{j}^{(r)} ; A_{j}\right)_{1, p}:\left(c_{j}^{(1)}, \gamma_{j}^{(1)} ; C_{j}^{(1)}\right)_{1, p_{1}} ; \ldots ;\left(c_{j}^{(r)}, \gamma_{j}^{(r)} ; C_{j}^{(r)}\right)_{1, p_{r}} \\
\vdots & \left(b_{j} ; \beta_{j}^{(1)}, \ldots, \beta_{j}^{(r)} ; B_{j}\right)_{1, q-1},(b ; 0, \ldots, 0 ; B):\left(d_{j}^{(1)}, \delta_{j}^{(1)} ; D_{j}^{(1)}\right)_{1, q_{1}} \ldots ;\left(d_{j}^{(r)}, \delta_{j}^{(r)} ; D_{j}^{(r)}\right)_{1, q_{r}}
\end{array}\right] \\
& =\frac{1}{z_{r}} \times \mathrm{I}_{p, q-1: p_{1}, q_{1} ; \ldots ; p_{r}, q_{r}}^{0, n m_{1}, n_{1} ; \ldots m_{r}, n_{r}}\left[\begin{array}{c|c}
z_{1} & \left(a_{j} ; \alpha_{j}^{(1)}, \ldots \alpha_{j}^{(r)} ; A_{j}\right)_{1, p}:\left(c_{j}^{(1)}, \gamma_{j}^{(1)} ; C_{j}^{(1)}\right)_{1, p_{1}} ; \ldots ;\left(c_{j}^{(r)}, \gamma_{j}^{(r)} ; C_{j}^{(r)}\right)_{1, p_{r}} \\
z_{r} & \left(b_{j} ; \beta_{j}^{(1)}, \ldots, \beta_{j}^{(r)} ; B_{j}\right)_{1, q-1}:\left(d_{j}^{(1)}, \delta_{j}^{(1)} ; D_{j}^{(1)}\right)_{1, q_{1}} ; \ldots ;\left(d_{j}^{(r)}, \delta_{j}^{(r)} ; D_{j}^{(r)}\right)_{1, q_{r}}
\end{array}\right],
\end{aligned}
$$

where $q-1 \geq 0, \mathfrak{R}(1-b)>0$,

(x)

$$
\begin{aligned}
& \mathrm{I}_{p, q: p_{1}, q_{1} ; \ldots ; p_{r}, q_{r}}^{0, n: m_{r}, n_{1} ; \ldots, m_{r_{1}}, n_{2}}\left[\begin{array}{c|l}
z_{1} & \left(a_{j} ; \alpha_{j}^{(1)}, \ldots \alpha_{j}^{(r)} ; A_{j}\right)_{1, p}:(c ; 0 ; C),\left(c_{j}^{(1)}, \gamma_{j}^{(1)} ; C_{j}^{(1)}\right)_{2, p_{1}} ; \ldots ;\left(c_{j}^{(r)}, \gamma_{j}^{(r)} ; C_{j}^{(r)}\right)_{1, p_{r}} \\
\vdots & \left(b_{j} ; \beta_{j}^{(1)}, \ldots, \beta_{j}^{(r)} ; B_{j}\right)_{1, q}:\left(d_{j}^{(1)}, \delta_{j}^{(1)} ; D_{j}^{(1)}\right)_{1, q_{1}} ; \ldots ;\left(d_{j}^{(r)}, \delta_{j}^{(r)} ; D_{j}^{(r)}\right)_{1, q_{r}}
\end{array}\right] \\
& =\Gamma^{C}(1-c) \times \mathrm{I}_{p, q: p_{1}-1, q_{1} ; \ldots ; p_{r}, q_{r}}^{0, n: m_{1}, n_{1}-1 ; \ldots ; m_{r}, n_{r}}\left[\begin{array}{c|c}
z_{1} & \left(a_{j} ; \alpha_{j}^{(1)}, \ldots \alpha_{j}^{(r)} ; A_{j}\right)_{1, p}:\left(c_{j}^{(1)}, \gamma_{j}^{(1)} ; C_{j}^{(1)}\right)_{2, p_{1}} ; \ldots ;\left(c_{j}^{(r)}, \gamma_{j}^{(r)} ; C_{j}^{(r)}\right)_{1, p_{r}} \\
\vdots & \left(b_{j} ; \beta_{j}^{(1)}, \ldots, \beta_{j}^{(r)} ; B_{j}\right)_{1, q}:\left(d_{j}^{(1)}, \delta_{j}^{(1)} ; D_{j}^{(1)}\right)_{1, q_{1}} ; \ldots ;\left(d_{j}^{(r)}, \delta_{j}^{(r)} ; D_{j}^{(r)}\right)_{1, q_{r}}
\end{array}\right],
\end{aligned}
$$

where $p_{1} \geq n_{1} \geq 1, \mathfrak{R}(1-c)>0$, 


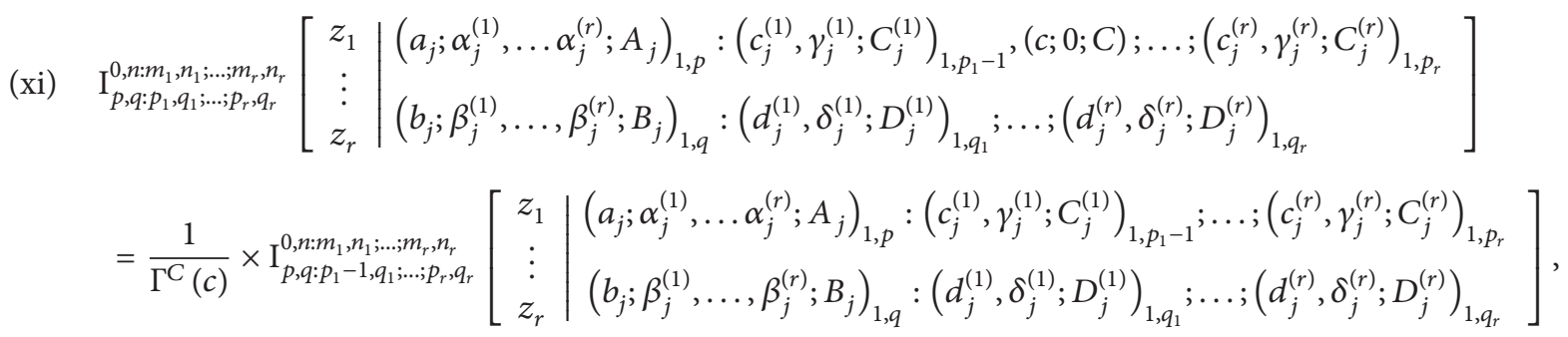

where $p_{1}-1 \geq n_{1} \geq 0, \Re(c)>0$,

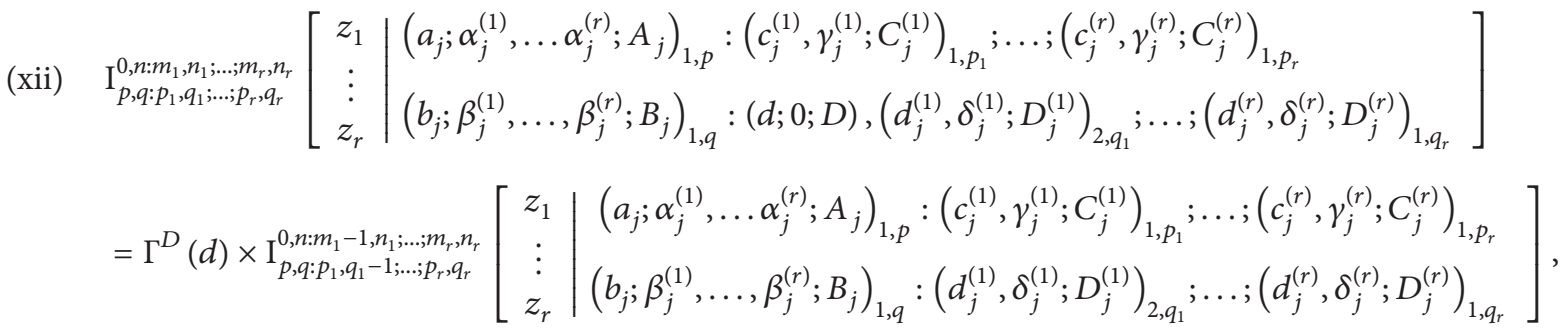

where $q_{1} \geq m_{1} \geq 1, \Re(d)>0$,

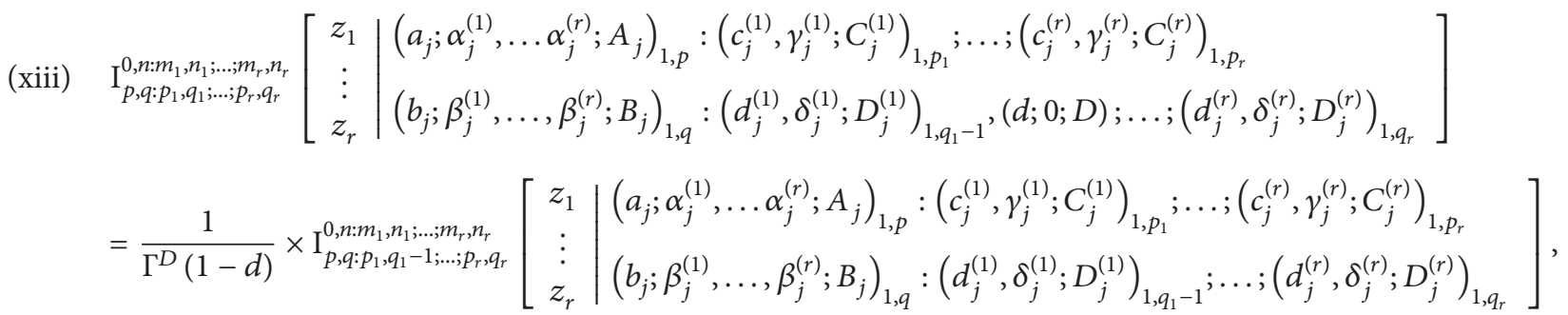

provided that $q_{1}-1 \geq m_{1} \geq 0, \Re(1-d) \geq 0$,

(xiv) $\quad \mathrm{I}_{p, q: p_{1}, q_{1} ; \ldots ; p_{r}, q_{r}}^{0, n m_{1}, n_{1} ; \ldots m_{r}, n_{r}}$

$$
\begin{aligned}
& {\left[\begin{array}{c|l}
z_{1} & \left(a_{j} ; \alpha_{j}^{(1)}, \alpha_{j}^{(r)} ; A_{j}\right)_{1, p}:\left(c_{j}^{(1)}, \gamma_{j}^{(1)} ; C_{j}^{(1)}\right)_{1, p_{1}} ; \ldots ;\left(c_{j}^{(r)}, \gamma_{j}^{(r)} ; C_{j}^{(r)}\right)_{1, p_{r}} \\
\vdots & \left(b_{j} ; \beta_{j}^{(1)}, \ldots, \beta_{j}^{(r)} ; B_{j}\right)_{1, q-1},\left(a_{1} ; \alpha_{1}^{(1)}, \ldots, \alpha_{1}^{(r)} ; A_{1}\right):\left(d_{j}^{1}, \delta_{j}^{(1)} ; D_{j}^{(1)}\right)_{1, q_{1}-1},\left(c_{1}^{(1)}, \gamma_{1}^{(1)} ; C_{1}^{(1)}\right) ; \ldots ;\left(d_{j}^{(r)}, \delta_{j}^{(r)} ; D_{j}^{(r)}\right)_{1, q_{r}-1},\left(c_{1}^{(r)}, \gamma_{1}^{(r)} ; C_{1}^{(r)}\right)
\end{array}\right]} \\
& =\mathrm{I}_{p-1, q-1: p_{1}-1, q_{1}-1 ; \ldots ; p_{r}-1, q_{r}-1}^{0, n-m_{1}, n_{1}-1 ; m_{r}, n_{r}-1}\left[\begin{array}{c|c}
z_{1} & \left(a_{j} ; \alpha_{j}^{(1)}, \ldots, \alpha_{j}^{(r)} ; A_{j}\right)_{2, p}:\left(c_{j}^{(1)}, \gamma_{j}^{(1)} ; C_{j}^{(1)}\right)_{2, p_{1}} ; \ldots ;\left(c_{j}^{(r)}, \gamma_{j}^{(r)} ; C_{j}^{(r)}\right)_{2, p_{r}} \\
\vdots & \left(b_{j} ; \beta_{j}^{(1)}, \beta_{j}^{(r)} ; B_{j}\right)_{1, q_{-1}}:\left(d_{j}^{(1)}, \delta_{j}^{(1)} ; D_{j}^{(1)}\right)_{1, q_{1}-1} ; \ldots ;\left(d_{j}^{(r)}, \delta_{j}^{(r)} ; D_{j}^{(r)}\right)_{1, q_{r}-1}
\end{array}\right],
\end{aligned}
$$

provided that $p \geq n \geq 1, p_{i} \geq n_{i} \geq 1, i=1, \ldots, r$, and $q \geq 1$,

$q_{i} \geq m_{i}+1, i=1, \ldots, r$, 
(xv)

$$
\begin{aligned}
& \mathrm{I}_{p, q: p_{1}, q_{1}, \ldots ; p_{r}, q_{r}}^{0, n: m_{1}, n_{r} ; ; m_{2}, n_{r}} \\
& {\left[\begin{array}{c|l}
z_{1} & \left.\left(a_{j} ; \alpha_{j}^{(1)}, \ldots, \alpha_{j}^{(r)} ; A_{j}\right)_{1, p}:\left(c_{j}^{(1)}, \gamma_{j}^{(1)} ; C_{j}^{(1)}\right)_{1, p_{1}-1},\left(d_{1}^{(1)}, \delta_{1}^{(1)} ; D_{1}^{(1)}\right) ; \ldots ;\left(c_{j}^{(r)}, \gamma_{j}^{(r)} ; C_{j}^{(r)}\right)_{1, p_{r}-1},\left(d_{1}^{(r)}, \delta_{1}^{(r)} ; D_{1}^{(r)}\right)\right] \\
\vdots & \left(b_{j} ; \beta_{j}^{(1)}, \beta_{j}^{(r)} ; B_{j}\right)_{1, q}:\left(d_{j}^{(1)}, \delta_{j}^{(1)} ; D_{j}^{(1)}\right)_{1, q_{1}} ; \ldots ;\left(d_{j}^{(r)}, \delta_{j}^{(r)} ; D_{j}^{(r)}\right)_{1, q_{r}}
\end{array}\right]} \\
& =\mathrm{I}_{p_{r}, q: p_{1}-1, q_{1}-1 ; \ldots ; p_{r}-1, q_{r}-1}^{0, n: m_{1}-1, n_{1} ; \ldots ; m_{r}-1, n_{r}}\left[\begin{array}{c|c}
z_{1} & \left(a_{j} ; \alpha_{j}^{(1)}, \ldots, \alpha_{j}^{(r)} ; A_{j}\right)_{1, p}:\left(c_{j}^{(1)}, \gamma_{j}^{(1)} ; C_{j}^{(1)}\right)_{1, p_{1}-1} ; \ldots ;\left(c_{j}^{(r)}, \gamma_{j}^{(r)} ; C_{j}^{(r)}\right)_{1, p_{r}-1} \\
z_{r} & \left(b_{j} ; \beta_{j}^{(1)}, \beta_{j}^{(r)} ; B_{j}\right)_{1, q}:\left(d_{j}^{(1)}, \delta_{j}^{(1)} ; D_{j}^{(1)}\right)_{2, q_{1}} ; \ldots ;\left(d_{j}^{(r)}, \delta_{j}^{(r)} ; D_{j}^{(r)}\right)_{2, q_{r}}
\end{array}\right],
\end{aligned}
$$

provided that $p \geq n+1, q \geq 1, p_{i} \geq n_{i}+1$, and $q_{i} \geq$ $m_{i} \geq 1, i=1, \ldots, r$,

(xvi)

$$
\mathrm{I}_{p, q: p_{1}, q_{1} ; \ldots ; p_{r}, q_{r}}^{0, n: m_{1}, n_{1} ; \ldots ; m_{r}, n_{r}}
$$

$$
\begin{aligned}
& {\left[\begin{array}{c|l}
z_{1} & \left(a_{j} ; \alpha_{j}^{(1)}, \ldots, \alpha_{j}^{(r)} ; A_{j}\right)_{1, p}:\left(c_{j}^{(1)}, \gamma_{j}^{(1)} ; C_{j}^{(1)}\right)_{1, p_{1}} ; \ldots ;\left(c_{j}^{(r)}, \gamma_{j}^{(r)} ; C_{j}^{(r)}\right)_{1, p_{r}} \\
\vdots & \left(b_{j} ; \beta_{j}^{(1)}, \ldots, \beta_{j}^{(r)} ; B_{j}\right)_{1, q}:\left(c_{p_{1}}^{(1)}, \gamma_{p_{1}}^{(1)} ; C_{p_{1}}^{(1)}\right),\left(d_{j}^{(1)}, \delta_{j}^{(1)} ; D_{j}^{(1)}\right)_{2, q_{1}} ; \ldots ;\left(c_{p_{r}}^{(r)}, \gamma_{p_{r}}^{(r)} ; C_{p_{r}}^{(r)}\right),\left(d_{j}^{(r)}, \delta_{j}^{(r)} ; D_{j}^{(r)}\right)_{2, q_{r}}
\end{array}\right]} \\
& =\mathrm{I}_{p, q:\left\{: m_{1}-1, n_{1} ; \ldots ; m_{r}-1, n_{r}\right.}^{0,1, q_{1}-1 ; \ldots ; p_{r}-1, q_{r}-1}\left[\begin{array}{c|l}
z_{1} & \left(a_{j} ; \alpha_{j}^{(1)}, \ldots, \alpha_{j}^{(r)} ; A_{j}\right)_{1, p}:\left(c_{j}^{(1)}, \gamma_{j}^{(1)} ; C_{j}^{(1)}\right)_{1, p_{1}-1} ; \ldots ;\left(c_{j}^{(r)}, \gamma_{j}^{(r)} ; C_{j}^{(r)}\right)_{1, p_{r}-1} \\
\vdots & \left(b_{j} ; \beta_{j}^{(1)}, \ldots, \beta_{j}^{(r)} ; B_{j}\right)_{1, q}:\left(d_{j}^{(1)}, \delta_{j}^{(1)} ; D_{j}^{(1)}\right)_{2, q_{1}} ; \ldots ;\left(d_{j}^{(r)}, \delta_{j}^{(r)} ; D_{j}^{(r)}\right)_{2, q_{r}}
\end{array}\right],
\end{aligned}
$$

provided that $p_{i} \geq n_{i}+1, q_{i} \geq m_{i}, i=1, \ldots, r$,

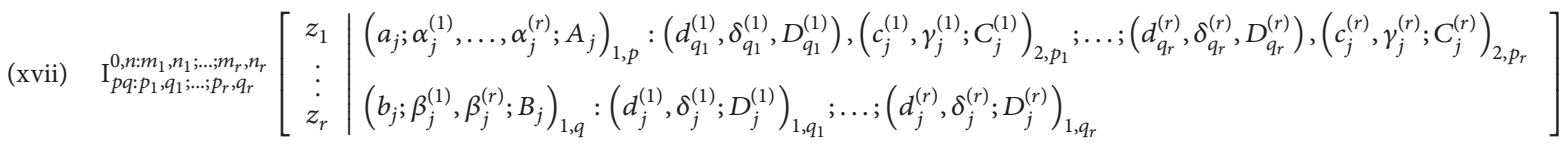

$$
\begin{aligned}
& =\mathrm{I}_{p, q: m_{1}, 1, q_{1}-1, \ldots ; \ldots ; p_{r}-1, q_{r}-1}^{0,1, \ldots, m_{r}, n_{r}-1}\left[\begin{array}{c|c}
z_{1} & \left(a_{j} ; \alpha_{j}^{(1)}, \ldots, \alpha_{j}^{(r)} ; A_{j}\right)_{1, p}:\left(c_{j}^{(1)}, \gamma_{j}^{(1)} ; C_{j}^{(1)}\right)_{2, p_{1}} ; \ldots ;\left(c_{j}^{(r)}, \gamma_{j}^{(r)} ; C_{j}^{(r)}\right)_{2, p_{r}} \\
\vdots & \left(b_{j} ; \beta_{j}^{(1)}, \beta_{j}^{(r)} ; B_{j}\right)_{1, q}:\left(d_{j}^{(1)}, \delta_{j}^{(1)} ; D_{j}^{(1)}\right)_{1, q_{1}-1} ; \ldots ;\left(d_{j}^{(r)}, \delta_{j}^{(r)} ; D_{j}^{(r)}\right)_{1, q_{r}-1}
\end{array}\right] \text {, }
\end{aligned}
$$

where $p \geq n, q \geq 1, p_{i} \geq n_{i} \geq 1, q_{i}-1 \geq m_{i}, i=1, \ldots, r$.

\section{Special Cases}

When $r=2$ and all the exponents $A_{j}(j=1, \ldots, p)$, $B_{j}(j=1, \ldots, q), C_{j}^{(i)}\left(j=1, \ldots, p_{i}, i=1 \ldots, r\right)$, and $D_{j}^{(i)}\left(1, \ldots, q_{i}, i=1 \ldots, r\right)$ the I-function of " $r$ " variables reduces to $\mathrm{H}$-function of two variables and therefore we obtain the corresponding results in $\mathrm{H}$-function of two variables [14].

\section{Conflict of Interests}

The authors declare that there is no conflict of interests regarding the publication of this paper.

\section{Acknowledgment}

The authors are immensely grateful to the worthy referee for some useful and valuable suggestions for the improvement of this paper which led to a better presentation. 


\section{References}

[1] A. K. Rathie, "A new generalization of generalized hypergeometric functions," Le Matematiche, vol. 52, no. 2, pp. 297-310, 1997.

[2] I. S. Ansari, F. Yilmaz, M. S. Alouni, and O. Kucur, "New results on the sum of Gamma random variates with application to the performance of wireless communication systems over nakagami-m fading channels," http://arxiv-web3.library.cornell .edu/abs/1202.2576v4.

[3] I. S. Ansari and Yilmaz, "On the sum of squared n-Random variates with application to the performance of wireless communication systems," http://arxiv-web3.library.cornell.edu/abs/1210 $.0100 \mathrm{vl}$.

[4] X. Minghua, W. Yik-Chung, and A. Sonia, "Exact outage probability of dual-hop CSI-assisted AF relaying over nakagami-m fading channels," IEEE Transactions on Signal Processing, vol. 60, no. 10, pp. 5578-5583, 2012.

[5] K. ShanthaKumari, T. M. Vasudevan Nambisan, and A. K. Rathie, "A study of the I-function of two variables," http://arxiv.org/abs/1212.6717 .

[6] P. K. Mittal and K. C. Gupta, "An integral involving generalized function of two variables," Proceedings of the Indian Academy of Sciences A, vol. 75, no. 3, pp. 117-123, 1972.

[7] H. M. Srivastava and R. Panda, "Some bilateral generating functions for a class of generalkized hypergeometric polynomials," Journal Für die Reine und Angewandte Mathematik, vol. 17, no. 288, pp. 265-274, 1976.

[8] B. L. J. Braaksma, "Asymptotic expansions and analytic continuations for a class of Barnesintegrals," Compositio Mathematicah, vol. 15, pp. 239-341, 1964.

[9] Y. L. Luke, The Special Functions and Their Approximations, vol. 1, Academic Press, New York, NY, USA, 1969.

[10] H. M. Srivastava and M. C. Daoust, "On Eulerian integrals associated with Kampé de Fériet's function," Publications De L'Institut Mathématique, vol. 9, no. 23, pp. 199-202, 1969.

[11] H. M. Srivastava and H. L. Manocha, A Treatise on Generating Functions, Halsted Press, Chichester, UK.

[12] E. M. Wright, "The asymptotic expansion of the generalized Bessel function," Proceedings of London Mathematical Society, vol. 38, pp. 257-270, 1935.

[13] A. Erdelyi, Higher Transcendental Functions, vol. 1, McGrawHill, New York, NY, USA, 1953.

[14] H. M. Srivastava, K. C. Gupta, and S. P. Goyal, The HFunctions of One and Two Variables With Applications, South Asian Publishers, New Delhi, India, 1982. 


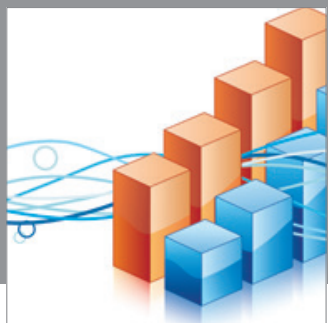

Advances in

Operations Research

mansans

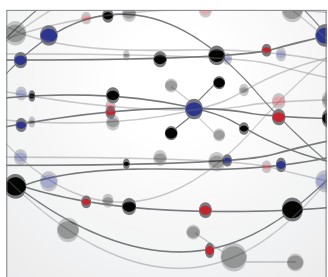

The Scientific World Journal
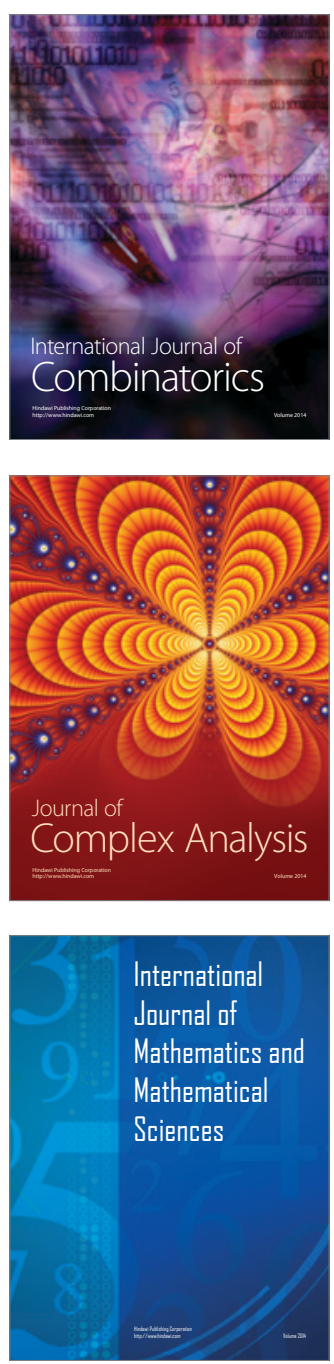
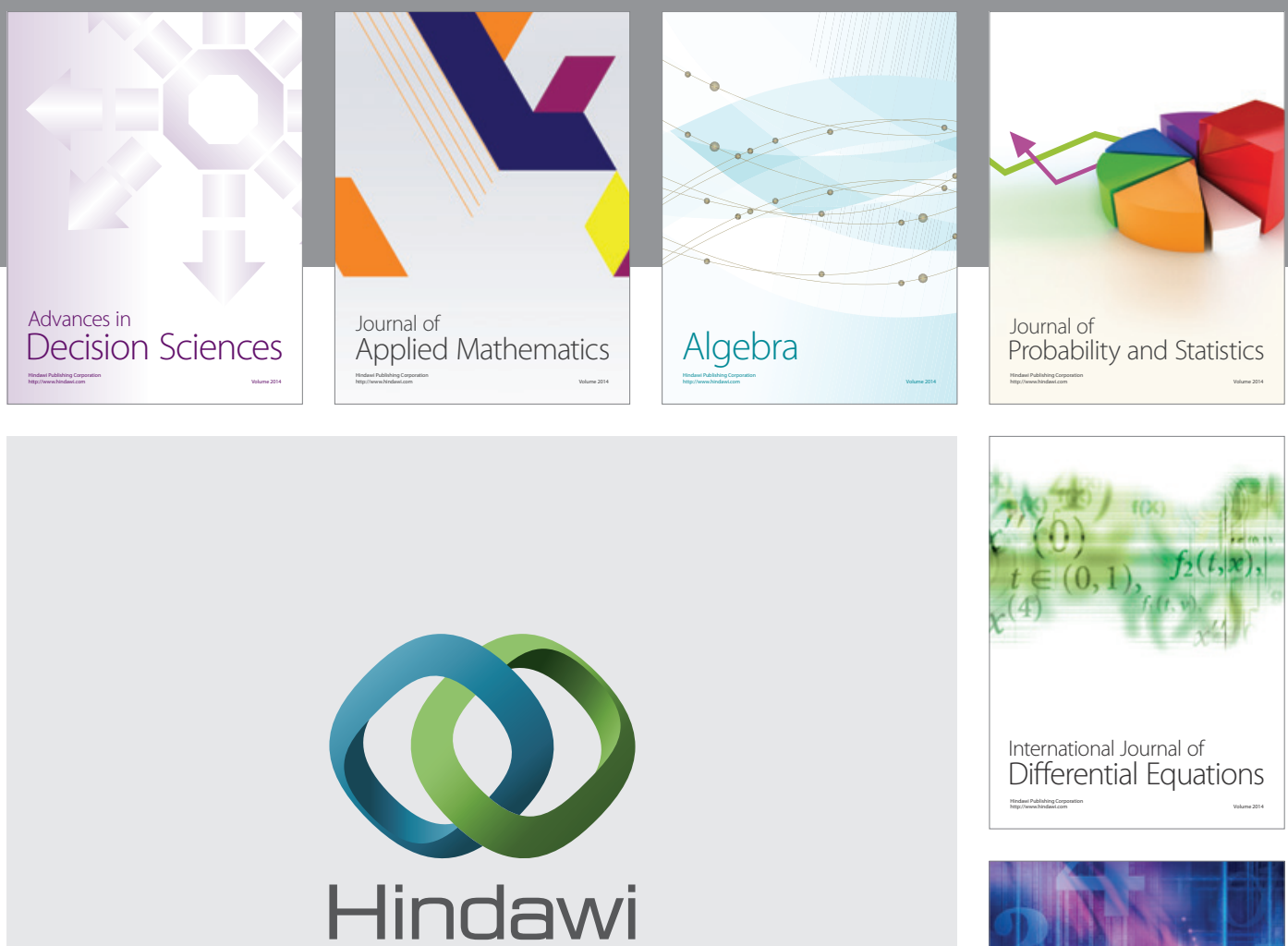

Submit your manuscripts at http://www.hindawi.com
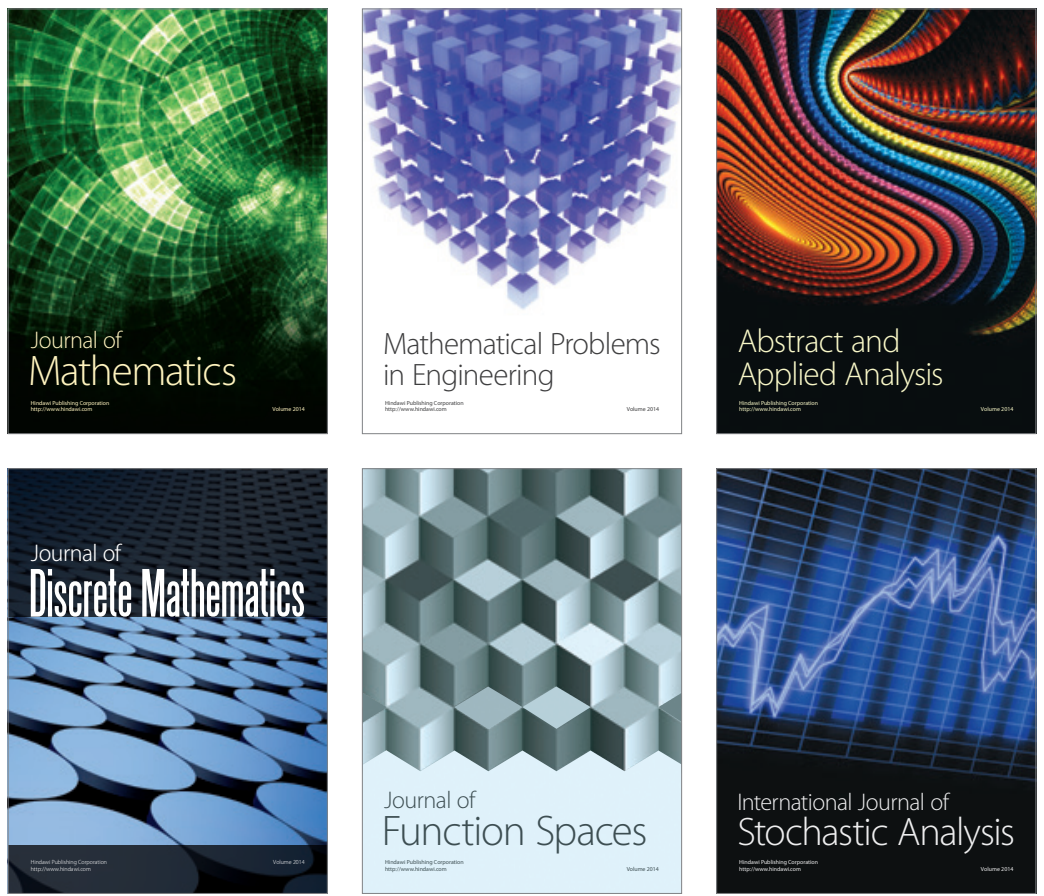

Journal of

Function Spaces

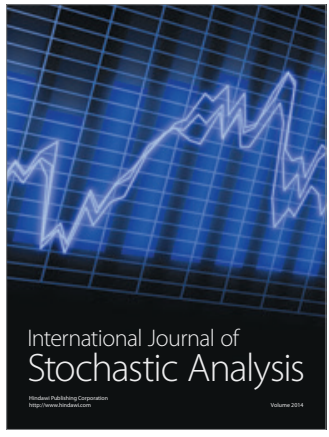

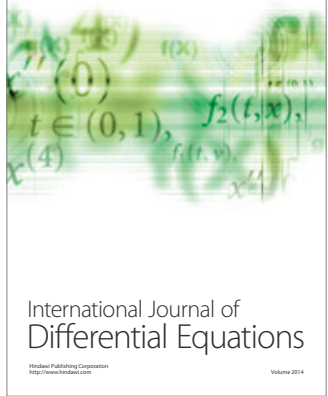
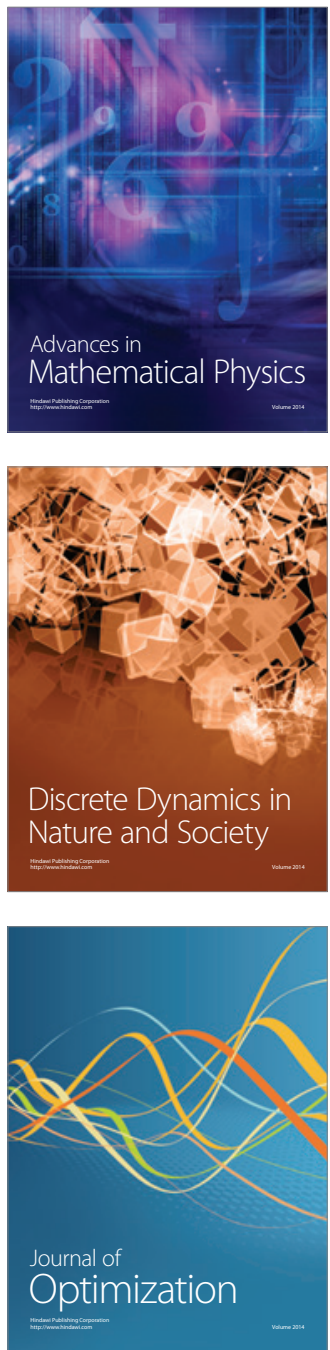